\title{
Elevated levels of plasma tumor necrosis factor alpha in patients with pseudoexfoliation glaucoma
}

This article was published in the following Dove Press journal:

Clinical Ophthalmology

\author{
Altaf A Kondkar' \\ Taif A Azad' \\ Faisal A Almobarak' \\ Hatem Kalantan' \\ Saleh A Al-Obeidan' \\ Khaled K Abu-Amero',2 \\ 'Glaucoma Research Chair, \\ Department of Ophthalmology, \\ College of Medicine, King Saud \\ University, Riyadh, Saudi Arabia; \\ ${ }^{2}$ Department of Ophthalmology and \\ Visual Sciences, University of Illinois \\ at Chicago, Chicago, IL, USA
}

Correspondence: Khaled K Abu-Amero Glaucoma Research Chair, Department of Ophthalmology, College of Medicine, King Saud University, PO Box 245, Riyadh II4II, Saudi Arabia

$\mathrm{Tel}+966$ I 2825290

Fax +966 | 4775724

Email abuamero@gmail.com
Background: Tumor necrosis factor alpha (TNF- $\alpha$ ) is a pro-inflammatory cytokine, which plays a role in glaucomatous neurodegeneration. Based on the plausible role of inflammation in the pathogenesis of pseudoexfoliation glaucoma (PEG), we investigated whether there is any relationship between the levels of plasma TNF- $\alpha$ and PEG or any of its clinical indices in comparison to normal controls.

Methods: The study was designed as a retrospective analysis. Plasma samples from 49 PEG patients and 88 non-glaucomatous controls were evaluated for TNF- $\alpha$ levels using an enzymelinked immunosorbent assay (ELISA). The assay was performed in duplicates on a biochemical/ ELISA analyzer.

Results: The two study groups were similar in age, sex and systemic disease distribution. The mean TNF- $\alpha$ concentration was significantly higher in the PEG patients $(5.54 \pm 4.58 \mathrm{pg} / \mathrm{mL})$ than in the control subjects $(0.93 \pm 1.49 \mathrm{pg} / \mathrm{mL} ; 95 \%$ confidence interval $[\mathrm{CI}]=3.50-5.72$; $p=0.000)$. The overall dose-response trend was significant $\left(\chi^{2}=57.07, \mathrm{df}=2 ; p=0.000\right)$. A moderate positive and significant correlation was seen between TNF- $\alpha$ level and cup/disc ratio, an important clinical index for PEG. Besides, binary logistic regression analysis showed that the risk of PEG was most significantly affected by TNF- $\alpha$ level as compared to no association with age and sex. In receiver operating characteristic analysis, the area under the curve was 0.777 $(95 \% \mathrm{CI}=0.682-0.872)$ and statistically significant $(p=0.000)$.

Conclusion: Elevated systemic levels of inflammatory marker, TNF- $\alpha$, are associated with PEG and may possibly serve as a biomarker for undiagnosed early glaucoma and/or as a marker for disease progression.

Keywords: glaucoma, inflammation, oxidative stress, pseudoexfoliation, TNF- $\alpha$

\section{Introduction}

Pseudoexfoliation syndrome (XFS) is a disorder of the extracellular matrix, which leads to deposition of an abnormal elastic microfibrillar material, primarily along the pupillary border, often producing a characteristic pattern on the anterior lens surface. ${ }^{1}$ Increased synthesis and/or decreased turnover of matrix components may be responsible for the excessive production and accumulation of abnormal matrix components. ${ }^{1}$ XFS may develop into pseudoexfoliation glaucoma (PEG), which is more aggressive and leads to worse prognosis than the more common form of glaucoma, (primary open angle glaucoma). A combination of clogging up of the trabeculum by pseudoexfoliation materials, pigments released from the iris, or both, as well as trabecular endothelial dysfunction could be a possible mechanism leading to the development of XFS and its subsequent progression to glaucoma (PEG). ${ }^{1,2}$

A characteristic feature of glaucoma is the death of retinal ganglion cells (RGCs). This is initiated at the occurrence of a pathological event, such as axonal injury, 
ischemia or changes in the lamina cribrosa, thereby activating apoptosis and increased oxidative stress leading to formation of free radicals at the localized tissues, which can initiate cytokine secretion in various inflammatory cells. ${ }^{3,4}$ These cytokines act as pro-inflammatory agents in the matrix process in XFS and PEG. ${ }^{5}$ In addition to glaucoma, altered chemokine and cytokine composition of the aqueous humor $(\mathrm{AH})$ has been observed in various ocular disease conditions including age-related macular degeneration, diabetic retinopathy and uveitis. ${ }^{6}$ Tumor necrosis factor-alpha (TNF- $\alpha$ ) is a pro-inflammatory cytokine playing an important role in various immunological and physiological processes. The diverse roles of TNF- $\alpha$ are mediated via two distinct receptors: a 55-kDa receptor 1 (TNF-R1) and a $75 \mathrm{kDa}$ receptor 2 (TNF-R2) and depends on which receptor is activated. ${ }^{7}$ The activation of TNF-R2 receptor is thought to be neuroprotective, and has been correlated with the presence of $\mathrm{Akt} /$ protein kinase $\mathrm{B}$ and involves phosphatidylinositol 3-kinase (PI3-kinase) signaling pathways. ${ }^{8}$ While activation of TNF-R1 receptors can activate inflammation in the tissue and apoptosis controlled by mitochondrial machinery. ${ }^{8}$ Overexpression of TNF- $\alpha$ can shift the balance and result in activation of TNF-R1 receptors. ${ }^{8}$ TNF- $\alpha$ and TNF-R1 mRNA were found to be upregulated in the retina of glaucoma patients, while anti-TNF- $\alpha$ antibodies were able to prevent RGC death in a mouse model of glaucoma. ${ }^{7}$ Both matrix metalloproteinases and tissue inhibitors of metalloproteinase expression are also affected by TNF- $\alpha$ level..$^{9,10}$ MMP-mediated extracellular matrix turnover and TIMPs regulate the activity of MMPs. An increased level of TNF- $\alpha$ might induce extra MMPs and/or TIMPs.

Various studies have demonstrated increased concentrations of TNF- $\alpha$ in glaucomatous aqueous humor. ${ }^{11,12}$ The exact role that these aqueous cytokines play in the pathogenesis of glaucoma is still unclear. Previous results have indicated that serum TNF- $\alpha$ was not altered in PEG patients compared to controls ${ }^{13}$ in contrast to other studies ${ }^{11,12}$ that found elevated levels of TNF- $\alpha$ in the AH of glaucoma patients. Inflammation is a common component of various age-related diseases, including PEG. Inflammatory biomarkers have the potential to become an important issue in clinical practice. Based on the role of inflammation in the pathogenesis of PEG, we investigated whether there is a difference in the levels of plasma TNF- $\alpha$ in PEG patients and controls.

\section{Materials and methods}

\section{Study population}

The study adhered to the tenets of the Declaration of Helsinki and was approved by the Institutional Review Board and
Research Ethics Committee of College of Medicine, King Saud University, Riyadh, Saudi Arabia (approval number \# 08-657). Informed consent was signed by all participating individuals. Saudi Arab participants with established clinically diagnosed PEG and ethnically matched healthy controls were recruited into the study at King Abdul-Aziz University Hospital in Riyadh, Saudi Arabia.

All the patients underwent a standardized detailed ophthalmic examination, which included measurement of intraocular pressure (IOP) by Goldmann applanation tonometry, slit lamp biomicroscopy, gonioscopy and dilated examination of the lens and fundus. Visual field was determined using the Humphrey automated field analyzer. The inclusion and exclusion criteria for patients and controls have been described previously. ${ }^{14}$ Subjects with PEG $(n=49)$ were defined as those with clinical evidence of exfoliation material on the pupil margin or anterior lens surface, the presence of glaucomatous optic neuropathy with associated visual field loss in one or both eyes, and documented IOP $\geq 22 \mathrm{mmHg}$ in either eye. Data on presence or absence of systemic diseases such as diabetes mellitus, hypertension, coronary artery disease and hypercholesterolemia, and smoking status were procured through their medical records and personal interviews. Exclusion criteria included a history of other possible optic neuropathies affecting either eye, significant visual loss in both eyes not associated with glaucoma, lack of adequate visualization of the fundus for disc assessment, history of steroid use, ocular trauma or refusal to participate. None of the participants included in this study were reported to have any infectious or immunomodulating diseases (rheumatoid arthritis, lupus, Crohn's disease). Saudi Arab subjects $(n=88)$ with no pseudoexfoliation material, healthy optic discs, normal anterior segments and normal IOP $(<18 \mathrm{mmHg})$ were recruited as controls. Subjects refusing to participate in this research study were excluded.

\section{Sample preparation and storage}

Blood samples from study subjects were collected in ethylenediaminetetraacetic acid tubes. The tubes were centrifuged at $5,500 \times g$ for $5 \mathrm{~min}$ and the plasma layer was separated and stored at $-80^{\circ} \mathrm{C}$ until use.

\section{ELISA measurement of TNF- $\alpha$ levels}

TNF- $\alpha$ concentrations were measured with an enzymelinked immunosorbent assay (ELISA; Catalog No STA00C, Quantikine ${ }^{\circledR}$ ELISA, R\&D Systems Inc., Minneapolis, MN, USA) in plasma samples from 49 PEG patients and 88 nonglaucomatous controls. The assay uses a monoclonal antibody specific for human TNF- $\alpha$ that has been pre-coated 
onto a microplate and was performed in duplicates on an automated biochemical analyzer, ChemWell-T (Awareness Technology Inc., Palm City, FL, USA), as per the manufacturer's instructions. The analyzer was programmed using a ChemWell-T Assay Editor in the standard ELISA assay mode to add $50 \mu \mathrm{L}$ of assay diluent and $200 \mu \mathrm{L}$ of plasma sample/standard and incubated for $2 \mathrm{~h}$ at room temperature (RT) with mixing for $1 \mathrm{~min}$. This was followed by washing (4× with soak time for $30 \mathrm{~s}$ ), addition of $200 \mu \mathrm{L}$ of human TNF- $\alpha$ conjugate and incubation for $2 \mathrm{~h}$ at RT with mixing for $1 \mathrm{~min}$. The washing step was repeated, followed by the addition of $200 \mu \mathrm{L}$ of substrates (equal volumes of stabilized hydrogen peroxide and tetramethylbenzidine; prepared immediately before use) and incubation at RT (in the dark) for $20 \mathrm{~min}$ with mixing for $1 \mathrm{~min}$. The reaction was terminated by the addition of $50 \mu \mathrm{L}$ stop solution and absorbance at $450 \mathrm{~nm}$ was measured. The concentration of TNF- $\alpha$ level was established utilizing the standard curve and expressed in $\mathrm{pg} / \mathrm{mL}$.

\section{Statistical analysis}

Data are presented as mean \pm SD for TNF- $\alpha$ concentration and other parameters. To analyze intergroup differences, Pearson's chi-square test was applied. Student $t$-test (2-tailed) was used to compare the TNF- $\alpha$ levels between normal and PEG samples. Mann-Whitney $U$ test (non-parametric) was used to compare median values between the patients and controls. Correlation testing was done using Spearman's rho test. A binary logistic regression analysis was performed to estimate the impact and direction of the effect of other risk factors and mean TNF- $\alpha$ level in patients with PEG. A receiver operating characteristic (ROC) curve was generated and the area under the curve (AUC) value was calculated by non-parametric Mann-Whitney $U$ statistics. A confidence interval (CI) was set to $95 \%$ and $p<0.05$ was considered statistically significant. Statistical analysis was performed with SPSS version 19.0 (IBM Corp., Armonk, NY, USA).

\section{Results}

In this study, we analyzed a total number of 137 subjects for TNF- $\alpha$ level that included 49 cases with PEG and 88 healthy normal controls. Table 1 presents various characteristics of the PEG cases and controls, including age, sex and TNF- $\alpha$ level. The results presented in Table 1, indicate that there was no significant difference in mean age $(p=0.159)$, sex $(p=0.909)$ and distribution of systemic diseases between the two groups. The mean TNF- $\alpha$ concentration was significantly higher in the PEG patients $(5.54 \pm 4.58 \mathrm{pg} / \mathrm{mL})$ than in the control subjects $(0.93 \pm 1.49 \mathrm{pg} / \mathrm{mL} ; 95 \% \mathrm{CI}=3.50-5.72 ; p=0.000)$.
Table I Distribution of demographic, clinical characteristics and TNF- $\alpha$ concentration in PEG cases and controls

\begin{tabular}{llll}
\hline Variables & $\begin{array}{l}\text { Cases } \\
(\mathbf{n}=\mathbf{4 9})\end{array}$ & $\begin{array}{l}\text { Controls } \\
(\mathbf{n}=\mathbf{8 8})\end{array}$ & $\begin{array}{l}\text { p-value } \\
\end{array}$ \\
\hline Age in years, mean (SD) & $59.8(6.6)$ & $57.1(12.1)$ & $0.159^{\mathrm{a}}$ \\
Sex (M/F) & $30 / 19$ & $53 / 35$ & $0.909^{\mathrm{b}}$ \\
Diabetes mellitus, n (\%) & $13(26.5)$ & $30(34.00)$ & $0.360^{\mathrm{b}}$ \\
Hypertension, n (\%) & $13(36.5)$ & $28(31.8)$ & $0.517^{\mathrm{b}}$ \\
Hypercholesterolemia, n (\%) & $\mathrm{I}(2.0)$ & $3(3.4)$ & $0.648^{\mathrm{b}}$ \\
Coronary artery disease, n (\%) & $0(0.0)$ & $2(3.4)$ & $0.287^{\mathrm{b}}$ \\
Smoking, n (\%) & $\mathrm{I}(2.0)$ & $4(4.5)$ & $0.129^{\mathrm{b}}$ \\
TNF- $\alpha$ in pg/mL, mean (SD) & $5.54(4.8)$ & $0.93(1.49)$ & $0.000^{\mathrm{a}}$ \\
TNF- $\alpha$ in $\mathrm{pg} / \mathrm{mL}$, median (range) & $5.19(0-17.8)$ & $0(0-8)$ & $0.000^{\mathrm{c}}$ \\
\hline
\end{tabular}

Notes: ${ }^{a}$ Independent sample $t$-test; 'Pearson's chi-square test; 'Mann-Whitney $U$ test.

Abbreviations: F, female; M, male; TNF- $\alpha$, tumor necrosis factor alpha; PEG, pseudoexfoliation glaucoma.

The median values, for TNF- $\alpha$ concentration, among the cases and the control subjects were $5.19 \mathrm{pg} / \mathrm{mL}$ (range $=0-17.8$ ) and $0 \mathrm{pg} / \mathrm{mL}$ (range $=0-8$ ), respectively and were also significantly different $(p=0.000)$ (Figure 1).

To assess the dose effect of TNF- $\alpha$ levels and the risk of PEG glaucoma, the levels of TNF- $\alpha$ among the cases and control subjects were initially dichotomized (uncategorized) at the 50th percentile (or median) value. Individuals with TNF- $\alpha$ levels $>0.5 \mathrm{pg} / \mathrm{mL}$ were at a statistically significant risk $(\mathrm{OR}=4.16,95 \% \mathrm{CI}=1.95-8.86$; $p=0.00017$; Table 2$)$. To assess any dose-response trend, the data were further dichotomized (uncategorized) into quartiles, and two cutoff points were identified at $0.0 \mathrm{pg} / \mathrm{mL}$ (first quartile or 25 th percentile) and $3.8 \mathrm{pg} / \mathrm{mL}$ (third quartile or 75 th percentile). Subjects were then categorized into three groups by using these two cutoff points. Overall, the dose-response trend

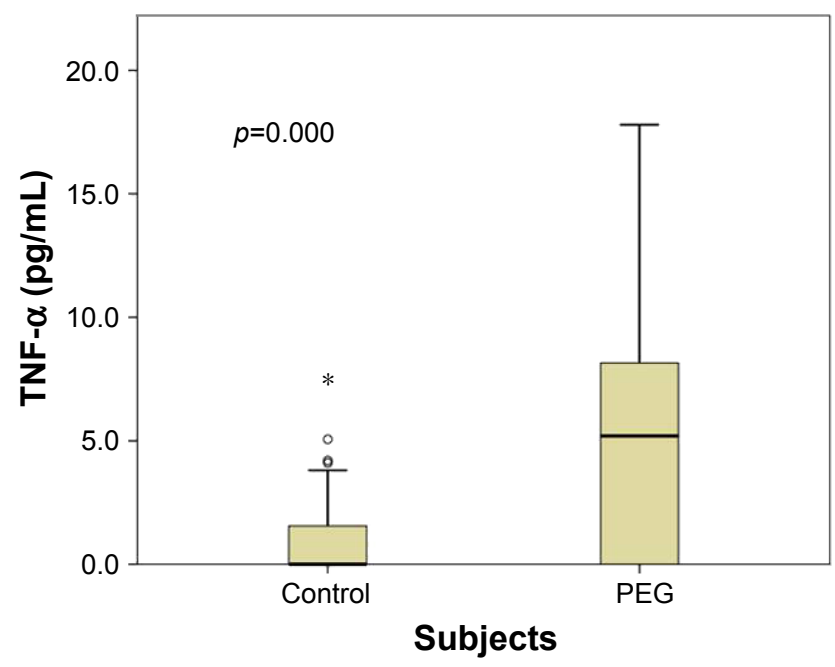

Figure I Box plot showing distribution of TNF- $\alpha$ level in PEG cases and control group.

Abbreviations: TNF- $\alpha$, tumor necrosis factor alpha; PEG, pseudoexfoliation glaucoma. 
Table 2 Dose effect of TNF- $\alpha$ level and the risk of PEG

\begin{tabular}{lllll}
\hline TNF- $\alpha$ & Cases & Controls & Odds & $95 \%$ confidence $p$-value \\
$(\mathrm{pg} / \mathrm{mL})$ & $(\mathrm{n}=49)$ & $(\mathrm{n}=88)$ & ratio & interval \\
& no $(\%)$ & no $(\%)$ & & \\
\hline
\end{tabular}

\begin{tabular}{|c|c|c|c|c|c|}
\hline \multicolumn{6}{|c|}{ By median } \\
\hline$\leq 0.5$ & $14(28.5)$ & $55(62.5)$ & Reference & - & - \\
\hline$>0.5$ & 35 (7I.4) & $33(37.5)$ & 4.16 & $1.95-8.86$ & 0.00017 \\
\hline \multicolumn{6}{|c|}{ By quartiles ${ }^{\mathrm{a}}$} \\
\hline 0 & 14 (28.5) & 51 (57.9) & Reference & - & - \\
\hline $0.1-3.8$ & $5(10.2)$ & $33(37.5)$ & 0.5 & $0.18-1.67$ & 0.31 \\
\hline$>3.8$ & $30(6 \mid .2)$ & $4(4.5)$ & 27.32 & $8.23-90.6$ & $1.08 \mathrm{e}-10$ \\
\hline
\end{tabular}

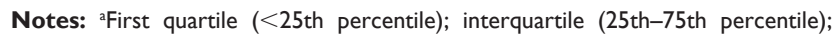
third quartile ( $>75$ th percentile). Overall dose response was significant $\left(\chi^{2}=57.07\right.$, $\mathrm{df}=2, p=0$ ).

Abbreviations: TNF- $\alpha$, tumor necrosis factor alpha; PEG, pseudoexfoliation glaucoma.

was statistically significant $\left(\chi^{2}=57.07, \mathrm{df}=2 ; p=0.000\right)$. Compared to individuals in which TNF- $\alpha$ concentration was absent/undetectable $(0 \mathrm{pg} / \mathrm{mL}$; first quartile), individuals in the medium category $(0.1-3.8 \mathrm{pg} / \mathrm{mL}$; interquartile) did not show any significant association; however, individuals in the highest category $(>3.8 \mathrm{pg} / \mathrm{mL}$; third quartile) were at a highly increased risk of the disease (OR $=27.32,95 \%$ CI $=8.23-90.6 ; p=1.08 \mathrm{e}-10$; Table 2).

A correlation testing between TNF- $\alpha$ levels and age and other clinical indices relevant to PEG such as cup/disc ratio, IOP and number of glaucoma medications was performed using Spearman's correlation. A moderate positive correlation of TNF- $\alpha$ level was seen with cup/disc ratio, but not with age, IOP and number of anti-glaucoma medications (Table 3). A logistic regression analysis was performed to evaluate the effect of age, sex and mean TNF- $\alpha$ level in patients with PEG using diseased/not diseased as the outcome (the dependent variable). The analysis showed that the risk of PEG was most significantly affected by TNF- $\alpha$ level as compared to no association with age and sex (Table 4).

In addition, to determine whether the levels of plasma TNF- $\alpha$ can be used to distinguish PEG patients and controls, we generated ROC curve to analyze the difference in the levels of plasma TNF- $\alpha$ between the two study groups.

Table 3 Correlation between TNF- $\alpha$ level and other clinical variables

\begin{tabular}{lll}
\hline Variables & $\boldsymbol{R}$ & $\boldsymbol{p}$-value \\
\hline Age & -0.049 & $0.57 \mathrm{I}$ \\
Intraocular pressure & -0.126 & 0.438 \\
Cup-to-disc ratio & 0.366 & 0.019 \\
Number of anti-glaucoma medications & 0.24 & 0.173 \\
\hline
\end{tabular}

Note: $R$, Spearman's correlation coefficient.

Abbreviation: PEG, pseudoexfoliation glaucoma.
Table 4 Regression analysis to determine the effect of age, sex and TNF- $\alpha$ levels on PEG

\begin{tabular}{llll}
\hline Variables & Odds ratio & $\begin{array}{l}\text { 95\% confidence } \\
\text { interval }\end{array}$ & -value \\
\hline Age & 1.045 & $0.996-1.096$ & 0.069 \\
Sex & 0.942 & $0.375-2.388$ & 0.899 \\
TNF- $\alpha$ & 1.632 & $1.364-1.952$ & 0.000 \\
\hline
\end{tabular}

Note: ${ }^{\mathrm{F} F e m a l e}$ as reference.

Abbreviations: TNF- $\alpha$, tumor necrosis factor alpha; PEG, pseudoexfoliation glaucoma.

Comparing PEG patients with controls, ROC curve area (AUC) was $0.777(95 \% \mathrm{CI}=0.682-0.872)$ and statistically significant $(p=0.000)$. These results indicate that the levels of plasma TNF- $\alpha$ can discriminate PEG patients with controls.

\section{Discussion}

The incidence of PEG varies across different populations and seems to be highest among individuals with Northern European and Scandinavian ancestry and lower among West Africans and African Americans. ${ }^{15,16}$ The prevalence of PEG in Saudi Arabia is unknown. ${ }^{17}$ Around 600 new glaucoma patients visit the Glaucoma Unit at King AbdulAziz University Hospital (where most PEG patients were recruited for this study) every year, and about $10 \%$ of those have PEG. The deposition of pseudoexfoliative material in iridocorneal angle with increased AH outflow resistance results in glaucoma. ${ }^{2}$ The cellular stress conditions such as ischemia, hypoxia, oxidative stress and inflammation play a critical role in the pathophysiology of XFS and PEG., ${ }^{4,5,14}$ This report demonstrates a clear association between elevated levels of plasma TNF- $\alpha$ and PEG.

Many studies have assessed the local and systemic status of TNF- $\alpha$ level in patients with XFS/PEG with conflicting findings. Sawada et al reported elevated levels of TNF- $\alpha$ in the AH of patients with PEG. ${ }^{11}$ Zenkel et al investigated expression of pro-inflammatory cytokines in eyes with early and late stages of XFS/PEG. ${ }^{5}$ The authors suggested a role for stress-induced subclinical inflammation in the onset of the fibrotic matrix process in XFS/PEG. ${ }^{5}$ However, there has been a study where aqueous levels of TNF- $\alpha$ were found not to be significant in PEG. ${ }^{18} \mathrm{~A}$ few studies have also evaluated systemic levels of TNF- $\alpha$ in XFS/PEG. Sarenac et al reported increased levels of aqueous TNF- $\alpha$ in patients with XFS and PEG but not in serum. ${ }^{13}$ By contrast, Sorkhabi et al has reported increased level of serum TNF- $\alpha$ to be risk a factor for systemic and ocular manifestations in patients with XFS (without glaucoma). ${ }^{19}$ Similarly, there have been 
investigations where systemic inflammatory markers such as serum alpha 1-antitrypsin activity, ${ }^{20}$ YKL-40, ${ }^{21}$ highsensitivity C-reactive protein ${ }^{19}$ and neutrophil-to-lymphocyte ratio $^{22}$ have been associated with XFS/PEG.

Most studies have utilized $\mathrm{AH}$ to estimate the level of TNF- $\alpha$ in glaucoma patients. Currently, there is no evidence directly correlating the level of cytokines in the eyes and serum/plasma. We estimated plasma level of TNF- $\alpha$ in welldefined PEG patients and compared to controls (no PEG or any ophthalmic disease as established by full ophthalmic examination). Age is an issue of particular importance to TNF- $\alpha$ level as age-related increases in TNF- $\alpha$ systemic levels could provide a basis for atherosclerosis, type diabetes and Alzheimer's disease in older individuals. ${ }^{23}$ Thus, our control group was carefully matched in terms of age, ethnicity, sex and other systemic conditions to the patient group. Mean TNF- $\alpha$ level was significantly higher in PEG patients and the effect was dose dependent, thus reinforcing our finding. Since there is no difference in systemic disease between the two study groups, the increase in TNF- $\alpha$ in the plasma could be more plausibly related to PFX rather than to one of the systemic diseases. The association of elevated TNF- $\alpha$ level with cup-to-disc ratio has clinical relevance as this is a fundamental parameter and a major clinical index for glaucoma. At present, we cannot offer a good explanation for any discrepancies between the TNF- $\alpha$ level in plasma and serum studies. Clearly, more investigations are needed at this end. However, the elevated level of TNF- $\alpha$ in the plasma (in this study) and the $\mathrm{AH}$ (previous studies) are clear indication of activated immune response against an inflammation.

Previous studies have shown that the cytokine TNF- $\alpha$ is capable of inducing experimental RGC death and optic neuropathies in human ${ }^{24}$ and animal models. ${ }^{25}$ In a mouse model of glaucoma, RGC and oligodendrocyte cell loss and consecutive optic atrophy was associated with upregulation of retinal TNF- $\alpha$ mRNA. ${ }^{25}$ Besides, exogenous administration of TNF- $\alpha$ was found to promote RGC loss and optic nerve degeneration, whereas genetic or pharmacological depletion of TNF- $\alpha$ or its receptors stimulated RGC survival. ${ }^{24}$ Furthermore, in vitro experiments have also revealed that TNF- $\alpha$ can induce RGC death through receptor-mediated caspase- 3 and caspase- 8 activation, and in a caspase-independent manner via the mitochondrial cell death pathway, which includes increased generation of free radical inducing oxidative damage. ${ }^{24}$ The fact that the mean TNF- $\alpha$ level was elevated in PEG patients compared to age-, sex- and ethnicity-matched healthy controls certainly indicates increased inflammation that may contribute to the creation of an oxidative stress status and, in a mechanism(s) as already described, such a situation may contribute to glaucoma pathogenesis.

The genetic basis of XFS/PEG is still not completely clear. To date, a number of genes have been linked to XFS, of which LOXL1 appears to be the most relevant in many populations, including Saudi Arabia, but accounts for only a small fraction of XFS/PEG cases. ${ }^{17}$ Besides, recent population-based genome-wide association studies have identified $C A C N A 1 A^{26}$ and five new susceptibility loci for XFS. ${ }^{27}$ Mutations in LOXL1 are expected to cause loss of elastin formation. As a result, there is loss of exfoliation material (XFM) from the anterior lens surface and disruption of the iris pigment epithelium, resulting in pigment deposition in the trabecular meshwork producing XFM locally. ${ }^{28}$ Thus, the mechanism by which LOXL1 PEG-risk SNPs may play a role in the development of PEG is quite different from that of the oxidative stress mechanism suggested here as a result of increased TNF- $\alpha$. Furthermore, a SNP (G-308A of rs1800629) upstream of the promoter site of TNF- $\alpha$ has been reported to cause elevation of TNF- $\alpha$ and is strongly associated with pseudoexfoliation syndrome in the Pakistani and Iranian populations. ${ }^{29,30}$ Similarly, there is some evidence for role of mitochondrial gene mutation in PEG patients in Saudi Arabia. ${ }^{31,32}$ A plausibility of similar mechanism(s) or as of yet unrecognized genetic/non-genetic interaction by elevated systemic levels of this marker contributing to glaucoma in our patients cannot be ruled out in this study.

The study has certain limitations. We acknowledge that the systemic increase in TNF- $\alpha$ level might not reflect the exact situation at the anterior segment structures, which are exposed to free radicals and thus more directly involved in the formation and development of glaucoma through the oxidative stress or other mechanism(s). This would require measurement of TNF- $\alpha$ level in AH samples. In addition, the study estimated only a single cytokine and the roles of other cytokines need to be investigated. Besides, the number of subjects evaluated, especially of patients with PEG, was small; therefore, the statistical power of the study may be limited.

\section{Conclusion}

In summary, we found elevated levels of plasma TNF- $\alpha$ in the serum of patients with PEG as compared to controls, indicating that the elevated systemic levels of this inflammatory marker may be associated with PEG. Despite the fact that there is no evidence supporting whether cytokine alterations in the serum or the plasma directly correlate with the cytokine levels in the eyes, elevated level of plasma TNF- $\alpha$ may serve 
as a possible biomarker for undiagnosed early glaucoma and/or as a marker for disease progression.

\section{Acknowledgments}

The authors would like to thank the Deanship of Scientific Research and Glaucoma Research Chair of the Department of Ophthalmology, College of Medicine, King Saud University for their support. However, it had no role in the design of the study and collection, analysis and interpretation of data and in writing the manuscript. The paper has not been presented in any previous conference or scientific meeting.

\section{Author contributions}

All authors contributed toward data analysis, drafting and revising the paper and agree to be accountable for all aspects of the work.

\section{Disclosure}

The authors report no conflicts of interest in this work.

\section{References}

1. Ritch R. Ocular and systemic manifestations of exfoliation syndrome. J Glaucoma. 2014;23(8 Suppl 1):S1-S8.

2. Ritch R, Schlötzer-Schrehardt U, Konstas AG. Why is glaucoma associated with exfoliation syndrome? Prog Retin Eye Res. 2003; 22(3):253-275.

3. Sahnoun Z, Jamoussi K, Zeghal KM. [Free radicals and antioxidants: physiology, human pathology and therapeutic aspects (part II)]. Therapie. 1998;53(4):315-339. French [with English abstract].

4. Nita M, Grzybowski A. The role of the reactive oxygen species and oxidative stress in the pathomechanism of the age-related ocular diseases and other pathologies of the anterior and posterior eye segments in adults. Oxid Med Cell Longev. 2016;2016:3164734.

5. Zenkel M, Lewczuk P, Jünemann A, Kruse FE, Naumann GO, Schlötzer-Schrehardt U. Proinflammatory cytokines are involved in the initiation of the abnormal matrix process in pseudoexfoliation syndrome/glaucoma. Am J Pathol. 2010;176(6):2868-2879.

6. Chua J, Vania M, Cheung CM, et al. Expression profile of inflammatory cytokines in aqueous from glaucomatous eyes. Mol Vis. 2012;18: 431-438.

7. Agarwal R, Agarwal P. Glaucomatous neurodegeneration: an eye on tumor necrosis factor-alpha. Indian J Ophthalmol. 2012;60(4):255-261.

8. Huang P, Zhang SS, Zhang C. The two sides of cytokine signaling and glaucomatous optic neuropathy. J Ocul Biol Dis Infor. 2009;2(2):78-83.

9. Meijer MJ, Mieremet-Ooms MA, van Hogezand RA, Lamers CB, Hommes DW, Verspaget HW. Role of matrix metalloproteinase, tissue inhibitor of metalloproteinase and tumor necrosis factor-alpha single nucleotide gene polymorphisms in inflammatory bowel disease. World J Gastroenterol. 2007;13(21):2960-2966.

10. Schlötzer-Schrehardt U, Lommatzsch J, Kuchle M, Konstas AG, Naumann GO. Matrix metalloproteinases and their inhibitors in aqueous humor of patients with pseudoexfoliation syndrome/glaucoma and primary open-angle glaucoma. Invest Ophthalmol Vis Sci. 2003; 44(3):1117-1125.

11. Sawada H, Fukuchi T, Tanaka T, Abe H. Tumor necrosis factor-alpha concentrations in the aqueous humor of patients with glaucoma. Invest Ophthalmol Vis Sci. 2010;51(2):903-906.
12. Balaiya S, Edwards J, Tillis T, Khetpal V, Chalam KV. Tumor necrosis factor-alpha (TNF-alpha) levels in aqueous humor of primary open angle glaucoma. Clin Ophthalmol. 2011;5:553-556.

13. Sarenac Vulovic TS, Pavlovic SM, Jakovljevic VL, Janicijevic KB, Zdravkovic NS. Nitric oxide and tumour necrosis factor alpha in the process of pseudoexfoliation glaucoma. Int J Ophthalmol. 2016;9(8): $1138-1142$.

14. Abu-Amero KK, Kondkar AA, Mousa A, Osman EA, Al-Obeidan SA. Decreased total antioxidants status in the plasma of patients with pseudoexfoliation glaucoma. Mol Vis. 2011;17:2769-2775.

15. Cashwell LF Jr, Shields MB. Exfoliation syndrome. Prevalence in a southeastern United States population. Arch Ophthalmol. 1988; 106(3):335-336.

16. Herndon LW, Challa P, Ababio-Danso B, et al. Survey of glaucoma in an eye clinic in Ghana, West Africa. J Glaucoma. 2002;11(5):421-425.

17. Abu-Amero KK, Osman EA, Dewedar AS, Schmidt S, Allingham RR, Al-Obeidan SA. Analysis of LOXL1 polymorphisms in a Saudi Arabian population with pseudoexfoliation glaucoma. Mol Vis. 2010;16: 2805-2810.

18. Takai Y, Tanito M, Ohira A. Multiplex cytokine analysis of aqueous humor in eyes with primary open-angle glaucoma, exfoliation glaucoma, and cataract. Invest Ophthalmol Vis Sci. 2012;53(1):241-247.

19. Sorkhabi R, Ghorbanihaghjo A, Ahoor M, Nahaei M, Rashtchizadeh N. High-sensitivity C-reactive protein and tumor necrosis factor alpha in pseudoexfoliation syndrome. Oman Med J. 2013;28(1):16-19.

20. Cumurcu T, Ozyurt H, Demir HD, Yardim H. Serum alpha-1-antitriypsin levels in patients with pseudoexfoliative syndrome. Curr Eye Res. 2008;33(2):159-162.

21. Türkyilmaz K, Öner V, Kirbas A, et al. Serum YKL-40 levels as a novel marker of inflammation and endothelial dysfunction in patients with pseudoexfoliation syndrome. Eye (Lond). 2013;27(7):854-859.

22. Kurtul BE, Ozer PA, Kabatas EU. Elevated neutrophil-to-lymphocyte ratio in pseudoexfoliation syndrome. Eye (Lond). 2016;30(8): $1045-1048$.

23. Brüünsgaard H, Pedersen BK. Age-related inflammatory cytokines and disease. Immunol Allergy Clin North Am. 2003;23(1):15-39.

24. Tezel G. TNF-alpha signaling in glaucomatous neurodegeneration. Prog Brain Res. 2008;173:409-421.

25. Nakazawa T, Nakazawa C, Matsubara A, et al. Tumor necrosis factoralpha mediates oligodendrocyte death and delayed retinal ganglion cell loss in a mouse model of glaucoma. $J$ Neurosci. 2006;26(49): 12633-12641.

26. Aung T, Ozaki M, Mizoguchi T, et al. A common variant mapping to CACNA1A is associated with susceptibility to exfoliation syndrome. Nat Genet. 2015;47(4):387-392.

27. Aung T, Ozaki M, Lee MC, et al. Genetic association study of exfoliation syndrome identifies a protective rare variant at LOXL1 and five new susceptibility loci. Nat Genet. 2017;49(7):993-1004.

28. Thorleifsson G, Magnusson KP, Sulem P, et al. Common sequence variants in the LOXL1 gene confer susceptibility to exfoliation glaucoma. Science. 2007;317(5843):1397-1400.

29. Khan MI, Micheal S, Rana N, et al. Association of tumor necrosis factor alpha gene polymorphism G-308A with pseudoexfoliative glaucoma in the Pakistani population. Mol Vis. 2009;15:2861-2867.

30. Razeghinejad MR, Rahat F, Kamali-Sarvestani E. Association of TNFA $-308 \mathrm{G} / \mathrm{A}$ and TNFRI $+36 \mathrm{~A} / \mathrm{G}$ gene polymorphisms with glaucoma. Ophthalmic Res. 2009;42(3):118-124.

31. Abu-Amero KK, Bosley TM, Morales J. Analysis of nuclear and mitochondrial genes in patients with pseudoexfoliation glaucoma. Mol Vis. 2008;14:29-36.

32. Abu-Amero KK, Cabrera VM, Larruga JM, Osman EA, Gonzalez AM, Al-Obeidan SA. Eurasian and Sub-Saharan African mitochondrial DNA haplogroup influences pseudoexfoliation glaucoma development in Saudi patients. Mol Vis. 2011;17:543-547. 
Clinical Ophthalmology

\section{Publish your work in this journal}

Clinical Ophthalmology is an international, peer-reviewed journal covering all subspecialties within ophthalmology. Key topics include: Optometry; Visual science; Pharmacology and drug therapy in eye diseases; Basic Sciences; Primary and Secondary eye care; Patien Safety and Quality of Care Improvements. This journal is indexed on

PubMed Central and CAS, and is the official journal of The Society of Clinical Ophthalmology (SCO). The manuscript management system is completely online and includes a very quick and fair peer-review system, which is all easy to use. Visit http://www.dovepress.com/ testimonials.php to read real quotes from published authors. 